\title{
Wirksamkeit von Entwicklungsklauseln in Chefarztverträgen Vorsicht bei vorformulierten Vertragsmustern
}

Sollte ein Krankenhaus Änderungen bezüglich der Abteilungsaufteilung, der Einstellung weiterer Ärzte, der Reduzierung der Bettenzahl oder Ähnliches planen, lohnt sich ein Blick in den zugrunde liegenden Dienstvertrag des Chefarztes. Sofern es sich um einen sogenannten „Formularvertrag“ handelt, unterliegt dieser einer strengen Wirksamkeitskontrolle. Ist eine pauschale Entwicklungsklausel enthalten, könnte dies die geplante Änderung insgesamt unwirksam machen. Gerade bei vorformulierten, häufig verwendeten Vertragsmustern sollte bei der Vertragsgestaltung daher besonderes Augenmerk auf das Recht der Allgemeinen Geschäftsbedingungen gelegt werden.

Chefarztverträge sehen häufig sogenannte „Entwicklungsklauseln“ vor, die es dem Krankenhausträger gestatten, Änderungen bezüglich der Abteilungsaufteilung, der Einstellung weiterer Ärzte, der Reduzierung der Bettenzahl oder Ähnliches vorzunehmen. In der Euphorie der Vertragsunterzeichnung wird eine solche Regelung von Seiten der zukünftigen Chefärzte häufig unterschätzt. Spätestens jedoch, wenn der Krankenhausträger ankündigt, von seinem Recht Gebrauch zu machen, stellen sich die Ärzte regelmäßig die Frage, ob dies denn überhaupt zulässig ist.

Das Arbeitsgericht Hagen hatte vor nicht allzu langer Zeit einen solchen Fall zu entscheiden (Urteil vom 05.09.2006, Aktenzeichen: 5 (2) Ca 2811/05). Erfreulicherweise kam das Gericht zu der Auffassung, dass auch Chefarztverträge sogenannte „Formulararbeitsverträge“ sein können, die einer wesentlich schärferen Kontrolle unterliegen.

\section{Der Fall:}

\section{Änderungsvorbehalt im Vertrag}

Der Kläger, ein Chefarzt, wurde von dem Krankenhausträger mit Dienstvertrag vom 01.07.2002 als „Leitender Arzt der Klinik für Allgemeine Chirurgie, Bereich Unfall- und Orthopädische Chirurgie des Krankenhauses“ angestellt. Vorausgegangen war eine Stellenausschreibung des Krankenhauses, in der es für seine Hauptfachabteilung „Allgemeine Chirurgie“ im Kollegialsystem zwei Chefärzte suchte. In dem vorgesehenen
Modell sollte ein Arzt den Bereich „Viszeralchirurgie“ und der andere Arzt den Bereich „Unfallchirurgie“ führen.

$\S 3$ Abs. 1 Nr. 1 des Dienstvertrages regelt eine Monatsvergütung in Anlehnung an die Vergütungsgruppe $1 \mathrm{der} \mathrm{Ar}$ beitsvertragsrichtlinien (AVR) der jeweils geltenden Fassung. Neben weiteren Regelungen fand sich folgende Klausel im Vertrag:

„Der Träger behält sich das Recht vor, jederzeit selbstständige Fachabteilungen, auch solche der gleichen Fachrichtung, oder Institute neu einzurichten oder abzutrennen und dafür weitere Abteilungsärzte einzustellen oder Belegärzte zuzulassen sowie neue Institutsleistungen zu erbringen. Er hat weiterhin das Recht, die Bettenzahl der Abteilungen zu ändern, Behandlungseinrichtungen zu ändern, aufzulösen oder neu einzurichten. Soweit der Arzt davon betroffen ist, ist er vorher zu hören. Bei diesen Maßnahmen ist ein Erstattungsanspruch des Arztes ausgeschlossen. Die Vergütung nach $\S 3$ Abs. 1 bleibt unverändert.“

Eine Zusatzvereinbarung zum Vertrag regelte, dass die im Krankenhaus „bestehende Klinik für Allgemeine Chirurgie ... ab dem 01.10.2002“ von dem Kläger und einem weiteren Arzt „gleichberechtigt im Kollegialsystem geleitet" wird.

\section{Kurzfristige Änderung:} zwei weitere leitende Ärzte

Nach einer Besprechung zwischen den Parteien im Jahr 2005 teilte das Kran- kenhaus dem Kläger mit einem Schreiben vom 26.09.2005 die „Aufstellung der neuen Abteilung für Unfallchirurgie“ mit. Auszugsweise lautete das Schreiben wie folgt:

„Der Feststellungsbescheid des Landes NRW vom 20.6.2005 ist nach Rücknahme von Widersprüchen verschiedener Nachbarkrankenhäuser nunmehr rechts- und bestandskräftig geworden. Wir verfügen jetzt über eine Subdisziplin Unfallchirurgie mit 25 Betten. Diese neue Abteilung muss sich nun schnellstmöglich am Markt etablieren und behaupten. Die zukünftigen Mindestmengen wie z.B. 50 Knie-TEPs ab 2006 müssen geleistet werden, damit die Unfallchirurgie auf Dauer gehalten werden kann. Verwaltungsrat und Geschäftsleitung sind übereinstimmend - aufgrund der Erfahrungen der letzten drei Jahre der Meinung, dass Sie dieser Herausforderung nicht gewachsen sein werden.

Aus diesem Grunde werden Sie zum 1.1.2006 Verstärkung durch zwei neue Kollegen erhalten, die zusammen mit Ihnen im Kollegialsystem die Abteilung leiten sollen. Die Leitung erfolgt im Kollegialsystem, wobei jedem Leitenden Arzt ein Teilgebiet mit eigener Verantwortung und eigenem Liquidationsrecht zugewiesen werden wird.

Es ist vorgesehen, dass Ihnen ausschließlich die Wirbelsäulenerkrankungen obliegen sollen. Dies umfasst die degenerativen und traumabedingten Erkrankungen der Wirbelsäule, wobei sowohl die konservativen wie auch operativen Behandlungen der Hals-, Brustund Lendenwirbelsäule ausschließlich durch Sie durchgeführt werden sollen. Die anderen Tätigkeitsfelder werden auf die beiden anderen Kollegen verteilt werden, wobei der eine für die elektive Endoprothetik und der andere für die klassische Unfallchirurgie einschließlich BG-Verfahren zuständig sein wird.

Grundlagen dieser Verteilung der Aufgaben und des Liquidationsrechts sind der 
gültige Feststellungsbescheid des Landes sowie die Entwicklungsklausel in Ihrem Arbeitsvertrag."

Diese Änderungen wollte der Chefarzt nicht akzeptieren. Er klagte unter anderem auf Feststellung der Unwirksamkeit der Änderung der Arbeitsbedingungen durch das Krankenhaus durch die Anweisung vom 26.09.2005.

\section{AGBs können auch für Chefarzt- verträge gelten}

Nach Ansicht des Arbeitsgerichts bestand der mit dem Chefarzt abgeschlossene Dienstvertrag aus vorformulierten Vertragsbedingungen: Der Vertrag stammte aus der von der Deutschen Krankenhausgesellschaft verfassten „Beratungs- und Formulierungshilfe Chefarzt-Vertrag“. Darüber hinaus wurde dieser Arbeitsvertrag im Krankenhaus standardgemäß verwendet. Unstrittig war auch, dass die Entwicklungsklausel nicht zwischen den Parteien „ausgehandelt" worden war. Das Gericht entschied daher, dass die vom Krankenhausträger für seine Anweisung vom 26.09.2005 als Rechtsgrundlage herangezogene Entwicklungsklausel einer Überprüfung nach den Maßstäben für vorformulierte Vertragsbedingungen nicht standhält. Grundlage der Gerichtsentscheidung ist das am 01.01.2002 in Kraft getretene Gesetz zur Modernisierung des Schuldrechts. Demnach erstreckt sich die Allgemeine-Geschäftsbedingungen-Kontrolle (AGB-Kontrolle) auch auf Formulararbeitsverträge. Entwicklungsklauseln in Chefarztverträgen unterliegen daher ebenfalls der AGB-rechtlichen Inhaltskontrolle.

\section{Änderungsvorbehalt muss \\ klar formuliert sein}

Die Entwicklungsklausel verstoße gegen diese Vorschriften des Bürgerlichen Gesetzbuches (BGB) und sei daher unwirksam, begründete das Gericht seine Entscheidung. In Allgemeinen Geschäftsbedingungen vereinbarte Änderungsvorbehalte - also die Vereinbarung eines Rechts des Verwenders (hier: der Krankenhausträger), die versprochene Leistung zu ändern oder von ihr abzuweichen - sind unwirksam, wenn nicht die Vereinbarung der Änderung oder Abweichung unter Berücksichtigung der Interessen des Verwenders für den anderen Vertragsteil (hier: der Chefarzt) zumutbar ist.
Im konkreten Fall wertete das Gericht die Beschäftigung des Arbeitnehmers zu den vereinbarten Bedingungen als die „versprochene Leistung“ durch den Arbeitgeber. Jeder Arbeitnehmer habe den Anspruch auf seine tatsächliche Beschäftigung, um seine Fähigkeiten zu erhalten und auszubauen sowie die in der Arbeit liegende Chance zur Entfaltung seiner Persönlichkeit zu nutzen.

Damit ein Widerrufsvorbehalt standhalten kann, müsste dieser möglichst konkret die Voraussetzungen festlegen, unter denen das einseitige Bestimmungsrecht entsteht und unter denen es auszuüben ist. Deshalb müsste der Arbeitnehmer bereits bei Vertragsschluss erkennen können, welche Leistungen die jeweilige Klausel erfasst und unter welchen Voraussetzungen ein Widerruf möglich werde.

\section{Mögliche Änderungsgründe angeben}

Bei den Voraussetzungen einer solchen Vertragsänderung, den Widerrufsgründen also, ließe sich zumindest die Richtung angeben, aus der der Widerruf möglich sein soll (wirtschaftliche Gründe, Leistung oder Verhalten des Arbeitnehmers), so das Gericht. Denn für den Arbeitnehmer sei es wichtig zu wissen, welcher Grad der Störung zugrunde gelegt werden soll, wie zum Beispiel

- die wirtschaftliche Notlage des Unternehmens

- das negative wirtschaftliche Ergebnis der Betriebsabteilung

- ein nicht ausreichender Gewinn

- der Rückgang bzw. das Nichterreichen der erwarteten wirtschaftlichen Entwicklung

- unterdurchschnittliche Leistungen des Arbeitnehmers

- schwerwiegende Pflichtverletzungen des Arbeitnehmers.

All dies müsste nach Auffassung des Gerichts konkretisiert werden, wenn der Arbeitgeber hierauf abstellen wolle. Allein allgemein auf die wirtschaftliche Entwicklung, die Leistungen oder das Verhalten des Arbeitnehmers gestützte Gründe sollen nicht ausreichen.

\section{Mangelnde Transparenz}

führt zur Unwirksamkeit

Insofern erweise sich die konkrete Entwicklungsklausel des Dienstvertrages
AGBs - im Alltag eher bekannt

Allgemeine Geschäftsbedingungen (AGBs), also vorformulierte Vertragsbedingungen, die für eine Vielzahl von Fällen angewendet werden, kennt der Laie eher im Zusammenhang mit Fitnessstudios oder Automietverträgen. Regelmäßig handelt es sich um das „Kleingedruckte“. Für derartige Verträge sieht das Bürgerliche Gesetzbuch (BGB) besonders strenge Voraussetzungen vor. Die Anwendung kann sich auch auf Chefarztverträge auswirken.

schon wegen der fehlenden Transparenz als unzumutbar und unwirksam. Die Entwicklungsklausel enthalte keinerlei Widerrufsgründe. Besondere Voraussetzungen oder Gründe für die Ausübung des erweiterten Direktionsrechts würden nicht genannt. Vielmehr solle das Krankenhaus das Recht haben, „jederzeit“ zum Beispiel selbstständige Fachabteilungen oder Institute neu einzurichten oder abzutrennen.

Ein solcher Änderungsvorbehalt sei jedoch nicht zumutbar, da er nicht klar und verständlich sei. Das Transparenzgebot werde nämlich auch dann verletzt, wenn nur eine beispielhafte Aufzählung der dem Krankenhausträger gestatteten organisatorischen Maßnahmen erfolge. Denn dann könne der Chefarzt nicht vorhersehen, mit welchen Maßnahmen er im Einzelfall rechnen müsse.

Dem Gericht lag offensichtlich auch daran, einmal klarzustellen, dass es kein Argument gebe, warum ein Änderungsvorbehalt bei Chefärzten eher zumutbar sein sollte als bei anderen Arbeitnehmern. Ein Chefarzt hat ein mindestens ebenso hohes Interesse daran, dass Leistungsänderungen zum Gegenstand von Verhandlungen gemacht und nicht einseitig in einer Weise durchgesetzt werden, die er bei Vertragsschluss nicht vorhersehen kann.

Im Ergebnis wurde also festgestellt, dass die Entwicklungsklausel unwirksam war, wohingegen der übrige Dienstvertrag wirksam blieb.

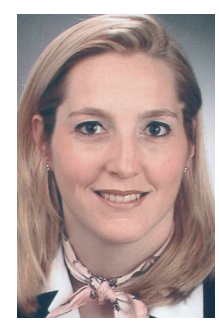

Korrespondenz Dr. iur. Isabel Häser Rechtsanwältin Ehlers, Ehlers \& Partner Widenmayerstraße 29 80538 München i.haeser@eep-law.de 